\title{
Beneficial effects of population bottlenecks in an RNA virus evolving at increased error rate
}

\author{
Clara Cases-González ${ }^{1}$, María Arribas ${ }^{1}$, Esteban Domingo ${ }^{1,2,3}$, and Ester Lázaro ${ }^{1 *}$ \\ ${ }^{1}$ Centro de Astrobiología (INTA-CSIC) Ctra de Ajalvir, Km 4, 28850 Madrid, Spain \\ ${ }^{2}$ Centro de Biología Molecular "Severo Ochoa" (CSIC-UAM) Campus de Cantoblanco, \\ 28049 Madrid, Spain \\ ${ }^{3}$ Centro de Investigación Biomédica en Red de Enfermedades Hepáticas y Digestivas \\ (CIBERehd) \\ *Corresponding Author: Ester Lázaro \\ lazarolm@inta.es \\ Tel: +34-915206457
}

Running Title: High error rate and population bottlenecks 


\section{Abstract}

RNA viruses replicate their genomes with a very high error rate and constitute highly heterogeneous mutant distributions similar to the molecular quasispecies introduced to explain the evolution of prebiotic replicators. The genetic information included in a quasispecies can only be faithfully transmitted below a critical error rate. When the error threshold is crossed the population structure disorganizes and it is substituted by a randomly distributed mutant spectrum. For viral quasispecies the increase of error rate is associated with a decrease in specific infectivity that can lead to the extinction of the population. In contrast, a strong resistance to extinction has been observed in populations subjected to bottleneck events despite the increased accumulation of mutations. In the present study we show that the mutagenic nucleoside analogue 5azacytidine (AZC) is a potent mutagen for bacteriophage $\mathrm{Q} \beta$. We have evaluated the effect of the increase in the replication error rate in populations of the bacteriophage $Q \beta$ evolving either in liquid medium, or during development of clonal populations in semisolid agar. Populations evolving in liquid medium in the presence of AZC were extinguished, while during plaque development in the presence of $\mathrm{AZC}$, the virus experienced a significant increase in the replicative ability. Individual viruses isolated from preextinction populations could withstand high error rates during a number of plaque-to-plaque transfers. The response to mutagenesis is interpreted in the light of features of plaque development versus infections by free-moving virus particles and the distance to a mutation-selection equilibrium. The results suggest that clonal bacteriophage populations away from equilibrium derive replicative benefits from increased mutation rates. This is relevant to the application of lethal mutagenesis in vivo, in the case of viruses that encounter changing environments, and are transmitted 
from cell to cell under conditions of limited diffusion that mimic the events taking place during plaque development.

Keywords: error rate, population bottlenecks, RNA viruses, mutagenesis, extinction 


\section{Introduction}

The high error rate of RNA virus replication, in the order of $10^{-3}$ to $10^{-5}$ misincorporations per nucleotide copied, ${ }^{1-4}$ confers RNA virus populations a high degree of genetic and phenotypic heterogeneity, which favors adaptability to changing and fluctuating environments. Molecular evolution theories, such as quasispecies theory, formulated in the context of the origin of life, predict that populations of genomes subjected to error-prone replication during sufficient time, under constant environmental conditions, can approach a stationary state characterized by a mutationselection equilibrium. ${ }^{5-7}$ This population structure is referred to as quasispecies, and consists of an ensemble of mutants represented at a constant frequency. ${ }^{8,9}$ RNA virus populations display features of quasispecies, although fluctuations in population size and in the physical and biological environmental conditions often place them away from equilibrium.

The stable transmission of the genetic information conveyed by a quasispecies can only take place below a limit of the replication error rate. ${ }^{10-12}$ When this limit, called the error threshold, is crossed the population structure disorganizes and it becomes a mutant spectrum randomly distributed through sequence space. ${ }^{6}$ Quasispecies theory predicts that this process implies an "error catastrophe", a phenomenon that in physics is described by a first order phase transition. ${ }^{11}$ Obviously, with real viruses a large expansion through sequence space can not occur, and several studies have documented that the increase of the error rate results in a decrease of specific infectivity, which can lead to the extinction of the population with modest expansion in sequence space ${ }^{13,14}$. The extinction of viruses through increased mutagenesis has been termed lethal mutagenesis, ${ }^{15-18}$ and constitutes a process whose molecular mechanisms are not well 
understood. Increased mutational pressure may prevent potentially beneficial mutations from being maintained in the population, and mutated genomes may exert an interfering effect on the replication of the viable genomes. ${ }^{14,19-21}$

The effects of population bottlenecks on virus extinction associated with lethal mutagenesis have not been examined. Population bottlenecks (the founding of a large virus population from one or a few parental genomes) imply a reduction in the genetic diversity that will be transmitted to the next generations. As a consequence of bottleneck events, mutations may be fixed at a higher rate, independently of their selective value, which can lead to progressive fitness reductions. ${ }^{22-27}$ Since the effects of most mutations on fitness depend on both the initial fitness and the genomic context, ${ }^{22,28}$ populations that have evolved through frequent bottlenecks can display variations in the frequency of advantageous, neutral and deleterious mutations. A number of studies carried out with RNA viruses, ${ }^{29}$ and in particular with several clones of foot-and-mouth disease virus (FMDV) subjected to successive plaque-to-plaque transfers, have shown that in the genomes surviving at late plaque transfers, some mutations can behave as compensatory mutations, reducing the deleterious effect of mutations fixed at earlier passages..$^{21,30-32}$

In the present study we have compared the effect of the increase of the replication error rate in large bacteriophage $\mathrm{Q} \beta$ populations adapted to replication in liquid culture medium with the effect in virus populations subjected to successive bottleneck events. We have chosen bacteriophage $\mathrm{Q} \beta$ as a model system because of the very large populations achieved, its long-term history of replication in Escherichia coli strains since its isolation, ${ }^{33,34}$ and its suitability to establish quasispecies dynamics with a 
calculated value for its mutation rate. ${ }^{1,34,35}$ Also, $Q \beta$-derived replicons have been used for quantitative measurements on mutation, competition and selection in vitro. ${ }^{36}$ Our results document that 5-azacytidine (AZC) is a potent mutagenic agent for bacteriophage $\mathrm{Q} \beta$, as previously shown for other RNA viruses. ${ }^{37,38}$ Complex bacteriophage $\mathrm{Q} \beta$ populations replicating in liquid medium in the presence of $\mathrm{AZC}$ were extinguished after a variable number of transfers. In contrast, AZC had no negative effects in the development of clonal populations of bacteriophage $\mathrm{Q} \beta$ in semisolid agar. AZC increased the replicative ability of the individual components of some virus clones that had experienced a large number of plaque-to-plaque transfers. Moreover, individual virus clones, when isolated from mutagenized populations that were doomed to extinction, could replicate under AZC mutagenesis during a number of plaque-to-plaque transfers. The results document an important effect of population bottlenecks in the response of viruses to lethal mutagenesis.

\section{Results}

Effect of AZC on the replication and genetic heterogeneity of complex populations of bacteriophage $Q \beta$ replicating in liquid culture medium

The effect of the mutagenic nucleoside analogue AZC in the replication of a heterogeneous population of bacteriophage $\mathrm{Q} \beta$ in E. coli was determined by passaging the virus in liquid medium in the presence of different AZC concentrations, as described in Materials and Methods. The phage, which already had an extremely long history of passages in E. coli, ${ }^{33,34}$ was further adapted to replication under laboratory culture conditions. The results (Fig. 1) show that replication in the presence of $40 \mu \mathrm{g} / \mathrm{ml} \mathrm{AZC}$ permits maintaining a viable phage population, albeit with orders of magnitude lower 
yields than the virus passaged in absence of AZC. This AZC concentration did not result in virus extinction after 16 serial transfers in the presence of mutagen. However, treatment with 80 or $120 \mu \mathrm{g} / \mathrm{ml}$ of AZC led to the loss of infectivity at transfer numbers 8 and 4 , respectively. To rescue any possible virus that could still persist after these transfers, 3 blind passages were carried out in the absence of mutagen. The inability to recover infectivity indicated that the virus population was extinguished. Supernatants of passages 3 (all populations), 6 (untreated control population, and populations treated with 40 and $80 \mu \mathrm{g} / \mathrm{ml}$ of AZC), and 13 (untreated control population, and population treated with $40 \mu \mathrm{g} / \mathrm{ml}$ of AZC) were plated on semisolid agar to isolate virus from 7 to 16 viral plaques. Viral RNA was extracted from each of them, amplified by RT-PCR, and sequenced. The results (Table 1) show an increase in the mutation frequency of viruses isolated from populations treated with $\mathrm{AZC}$, relative to populations that were not treated with mutagen. However, the increase was only significant for populations treated with low doses of mutagen for a limited number of passages. Thus, AZC is mutagenic for bacteriophage $\mathrm{Q} \beta$, and a complex bacteriophage $\mathrm{Q} \beta$ population was extinguished upon treatment with it. The loss of infectivity was not associated with the highest value of the mutation frequency, determined with biological clones of the corresponding populations.

Effect of AZC on the replication and genetic heterogeneity of clonal populations of bacteriophage $Q \beta$ replicating within lytic plaques

The initial Q $\beta$ population used for the passages in the presence of AZC (Fig. 1) was diluted and plated in semisolid agar medium, and virus from 4 biological clones, named $\mathrm{C} 1 \mathrm{pt} 1, \mathrm{C} 2 \mathrm{pt} 1, \mathrm{C} 3 \mathrm{pt} 1$, and $\mathrm{C} 4 \mathrm{pt} 1$ was obtained. This nomenclature indicates the virus 
clone and the plaque transfer (pt) at which it was isolated. Two series of 50 and 40 plaque-to-plaque transfers were initiated with clones $\mathrm{C} 3 \mathrm{pt} 1$ and $\mathrm{C} 4 \mathrm{pt} 1$, respectively. The variation of the virus replicative ability was estimated by determining the virus titer in plaques obtained at each transfer (Fig. 2). Although plaque titers in the C4pt1 transfer series decreased at a higher rate than in the C3pt1 series (Fig. 2), the consensus genomic nucleotide sequence (from nucleotide 250 to 4000) revealed the accumulation of a similar number of mutations in the two lineages ( 9 mutations for C3pt40 and 8 mutations for C4pt40) (Table 2).

The effect of $\mathrm{AZC}$ on $\mathrm{Q} \beta$ clonal populations was assayed with clones $\mathrm{C} 1 \mathrm{pt} 1$ and $\mathrm{C} 2 \mathrm{pt} 1$ and with two clones isolated after a large number of plaque-to-plaque transfers (C3pt38 and $\mathrm{C} 4 \mathrm{pt} 40$ ). The 4 virus clones were plated either in the presence or the absence of AZC $(120 \mu \mathrm{g} / \mathrm{ml})$, and subclones were isolated. The titer of the subclones obtained in the absence of AZC was taken as an estimate of the replicative ability of the individual viruses composing the parental clonal populations. The comparison of the titers in plaques obtained in the presence and the absence of AZC was used to evaluate whether AZC had some influence in the replicative ability of the subclonal components of the clonal viruses.

The average titer of the C1pt1 and C2pt1 subclones was 9- and 4-fold higher, respectively, than the titer of the corresponding parental clones, and titers were similar when subclones were developed in the presence or the absence of AZC $(p>0.05$, Student's $t$ test) (Fig. 3). While C3pt38 subclones isolated in the absence of AZC had an average titer 8-fold lower than its parental clone, C4pt40 subclones exhibited a 3-fold increase in the average titer relative to the parental clone (Fig. 3). However, both 
$\mathrm{C} 3 \mathrm{pt} 38$ and $\mathrm{C} 4 \mathrm{pt} 40$ clones, when plated in presence of $\mathrm{AZC}(120 \mu \mathrm{g} / \mathrm{ml})$, gave rise to subclones that showed a significant increase in the average titer relative to that obtained in the absence of AZC (Fig. 3). These results indicate that AZC had no detectable effect on virus replication within lytic plaques founded by viruses not subjected to plaque-toplaque transfers, but AZC had a beneficial effect on the development of plaques founded by viruses subjected to a large number of plaque-to-plaque transfers.

The results suggest that $\mathrm{C} 3 \mathrm{pt} 38$ and $\mathrm{C} 4 \mathrm{pt} 40$ clones were composed of individual viruses able to originate a larger amount of infectious progeny when the error rate increased. However, the result did not clarify whether this increase was due to the generation of a higher average number of beneficial mutations that favored faster replication, or it was a consequence of some influence of AZC on cellular or viral processes critical for plaque development. If the latter possibility were true, the effect of AZC would be dependent on its continuous presence in the culture medium. To address this question we selected subclone C4pt41.11 (developed in presence of AZC), which had a higher titer than the average for $\mathrm{C} 4 \mathrm{pt} 40$ subclones developed in the absence of AZC (Fig. 4, right part). C4pt41.11 was plated in the absence of $\mathrm{AZC}$, and lytic plaques were isolated and titrated. Although the average titer of the progeny plaques experienced an 8-fold decrease relative to $\mathrm{C} 4 \mathrm{pt} 41.11$, it was still significantly higher than the average titer of C4pt40 subclones developed in the absence of AZC ( $p<0.05$, Student's $t$ test). The result suggests that the beneficial effect of AZC persists at least after an additional plaque transfer in the absence of AZC.

The effect of AZC on the replicative ability of clonal populations of bacteriophage $Q \beta$ is related to its mutagenic action 
To investigate whether the increase in titer observed when plaque development took place in the presence of AZC could be due to the unspecific accumulation of any nucleoside derivative, we determined the average titer of a number of subclones derived from C4pt41.6 (a C4pt40 subclone isolated in the absence of AZC) either in the presence or absence of $\mathrm{AZC}(120 \mu \mathrm{g} / \mathrm{ml})$, or in the presence of the same molar concentration of cytidine (the non mutagenic standard nucleoside to which AZC is an analogue) (Fig. 4, left part). Subclones developed in presence of AZC had a statistically significant higher titer than those developed in the absence of AZC or in the presence of cytidine ( $p<0.05$, Student's $t$ test). In contrast, the average titer of subclones developed in the presence of cytidine did not differ significantly from the titer obtained in the absence of nucleosides $(p>0.05$, Student's $t$ test). This difference suggests that the increase in titer observed when plaque development occurs in the presence of AZC might be directly related to its mutagenic action.

The effect of AZC on the viral genome was evaluated by comparing the average mutation frequency of individual clonal populations grown in the presence or the absence of AZC. To this aim, two plaques derived from C4pt40, one developed in the presence of $\mathrm{AZC}$ and the other one in its absence, were plated to obtain subclones whose RNA was subjected to RT-PCR and nucleotide sequencing (genomic residues 1407 to 2817). The average mutation frequency observed in 18 subclones derived from the plaque developed in the presence of AZC was $2 \times 10^{-4}$ substitutions per nucleotide $(\mathrm{s} / \mathrm{nt})$, whereas the average mutation frequency determined in 20 subclones derived from the plaque developed without AZC was $4 \times 10^{-5} \mathrm{~s} / \mathrm{nt}$. Although this difference is not 
statistically significant ( $p=0.07$, Student's $t$ test), it suggests a relationship between the effect of AZC on plaque development and its mutagenic action.

Extinction of virus infectivity in liquid medium versus sustained replication through plaque-to-plaque transfers in the presence of AZC.

The extinction of bacteriophage $\mathrm{Q} \beta$ in the presence of $\mathrm{AZC}$ in liquid medium and its survival and increase in titer when replication took place in semisolid agar, was further investigated. Two low titer subclones (C4pt41.16 and $\mathrm{C} 4 \mathrm{pt} 41.17)$, both derived from $\mathrm{C} 4 \mathrm{pt} 40$ in the absence of $\mathrm{AZC}$, were used to infect $E$. coli either in liquid medium or in semisolid agar at a very low multiplicity of infection $\left[\mathrm{MOI} \sim 10^{-7}\right.$ plaque forming units (pfu) per bacterium]. After 3 serial transfers in liquid medium in the presence of AZC $(120 \mu \mathrm{g} / \mathrm{ml})$ both populations were extinguished, while they survived during several plaque-to-plaque transfers in the presence of the same AZC concentration (Fig. 5).

To analyze whether the viruses composing preextinction populations were unavoidably destined to extinction, an aliquot of two preextinction populations -obtained after subjecting subclones $\mathrm{C} 4 \mathrm{pt} 41.3$ and $\mathrm{C} 4 \mathrm{pt} 41.17$ (Fig. 6) to 2 passages in liquid medium with AZC (120 $\mu \mathrm{g} / \mathrm{ml})$ - was plated in the presence of AZC (120 $\mu \mathrm{g} / \mathrm{ml})$, and subjected to 2 additional plaque-to-plaque transfers in the presence of the mutagen. Both clones survived without significant fitness loss (Fig. 6). Thus, preextinction populations can contain individual viruses that can replicate and be maintained in semisolid agar in the presence of AZC. Therefore, a mutagenic activity can have either a deleterious effect or provide a survival-enhancement force on a viral genome, depending on its evolutionary history. 


\section{Discussion}

There is extensive experimental evidence that RNA virus replication is placed very close to an error threshold, and that modest increases of the mutation rate above the threshold have a negative impact on the viability of viral populations. ${ }^{12,15,16,18,38-40}$ In contrast to the frequent extinctions reported in virus populations in which the error rate has been increased through the use of mutagens, a strong resistance to extinction at the population level has been documented in virus populations subjected to a large number of plaque-to-plaque transfers. ${ }^{21,30,41}$ Resistance to extinction of viruses subjected to repeated population bottlenecks is observed despite fitness loss and a linear accumulation of mutations that reaches higher levels than those scored in mutant spectra of preextinction populations. ${ }^{21}$ Hence, the consequences of the increase of the error rate in RNA virus populations subjected to bottleneck events deserve being explored in detail and it has been addressed in the present study.

In agreement with results obtained with other RNA viruses, ${ }^{16-18}$ populations of bacteriophage $\mathrm{Q} \beta$ were extinguished following a low number of serial infections in liquid medium, in the presence of the mutagenic nucleoside analogue AZC. The kinetics of the process is characterized by a decrease in the virus titer as the transfer number increases, accompanied of an increase in mutation frequency (Fig. 1 and Table 1). Since viruses that have accumulated a large number of mutations possibly cannot give rise to lytic plaques, the mutation frequency reached a maximum value at a certain passage, without further increase as the population approached extinction. This behavior probably reflects some limit in the number of mutations compatible with plaque formation. In contrast, clonal populations developed in semisolid agar in the presence of 
AZC did not exhibit any negative effect regarding the replicative ability of their components (Fig. 3). The same virus clones that could be maintained replicating through plaque-to-plaque transfers in the presence of $\mathrm{AZC}$ were extinguished in infections in liquid medium (Fig. 5). Clonal populations rescued from preextinction populations in liquid medium, maintained viability in the presence of $\mathrm{AZC}$ during a number of plaque-to-plaque transfers (Fig. 6). These contrasting results strongly suggest that the effect of AZC on virus replication is not due to some general alteration of host metabolism since the latter should have influenced virus replication both in liquid culture medium and in semisolid agar medium.

Several factors could influence the disparate consequences that the increase of error rate has in populations evolving in liquid medium or within lytic plaques in semisolid agar medium. One influence could be the multiplicity of infection (MOI). In liquid culture medium the initial MOI was maintained around $0.1 \mathrm{pfu}$ per cell and could not reach significantly higher values than 10 pfu per cell (Fig. 1). In contrast, the burst size of an E.coli cell infected with bacteriophage $\mathrm{Q} \beta$ [about 2000 pfu per cell ${ }^{42}$ ] should ensure a very high MOI during plaque development. Another possible influence is the complexity of the mutant spectrum generated during infections in liquid medium versus during plaque development. The presence at high error rate of a complex mutant spectrum, composed by defective and highly mutated genomes, which could interfere with the replication of viable viruses, has been proposed as a pathway towards virus extinction. ${ }^{14,43,44}$ While interfering genomes can be generated either in infections in liquid culture medium or in semisolid agar medium, plaque-to-plaque transfers permit at each transfer to purge the virus population of defective or low fitness mutants generated at the previous transfer. This should favor the sustained replication of viruses in the 
presence of $\mathrm{AZC}$, even when they have been isolated from preextinction populations (Figs. 5 and 6).

Infections in liquid medium and in semisolid agar differ also in virus diffusion, which is much more limited in the semisolid medium. ${ }^{45}$ Limited diffusion can restrict virus accessibility to the host cells. ${ }^{46}$ Therefore, a fraction of the progeny viruses cannot initiate new infections and remain in the plaque without accumulating additional mutations, a fact that can be beneficial when the error rate increases. Careful analysis of lytic plaques of bacteriophage $\mathrm{T} 7$ showed that the frequency of mutants relative to the founder virus increased with the distance to the centre of the plaque, which was occupied by a larger amount of non-mutated genomes than the plaque periphery. ${ }^{47}$ Therefore, the consequences of the increase of the error rate during plaque development can be attenuated by a subclass of mutants that have undergone a low number of replication rounds and have accumulated a low number of mutations.

The effect of AZC in the titer of subclones generated upon replication of the viruses contained in different lytic plaques varied with their previous history of plaque-toplaque transfers. The virus titer measured within a plaque will depend on the fitness of the founder virus and on the type of mutants, deleterious or beneficial, which it generates in a given genomic context. ${ }^{22,48,49}$ However, the effect of AZC on Q $\beta$ clones did not depend on the initial viral fitness, as demonstrated by the differences observed between viruses that produced the same progeny titer (compare results for $\mathrm{C} 1 \mathrm{pt} 1$ and $\mathrm{C} 2 \mathrm{pt} 1$ with results for $\mathrm{C} 3 \mathrm{pt} 38$ in Fig. 3). These viruses differed in the number of plaqueto-plaque transfers and in the genomic context in which mutation occurred. Replication of clones $\mathrm{C} 1 \mathrm{pt} 1$ and $\mathrm{C} 2 \mathrm{pt} 1$, which were mostly composed by viruses with higher 
replicative ability than the parental virus (see subclone titers in the absence of AZC in Fig. 3), was not affected by AZC. In contrast, the individual viruses composing C4pt40 clone, which also presented higher replicative ability than the parental virus, experienced a significant increase of the replicative ability in the presence of AZC. C3pt38 subclones, which displayed lower average replicative ability than the parental virus, also experienced a significant fitness increase when they were developed in the presence of AZC. These results exclude high MOI during plaque development as a significant influence on the advantage of replication in the presence of AZC. Rather, the results suggest that the effect of $\mathrm{AZC}$ depends on the proximity of the clonal viruses to the heterogeneous population that started the plaque-to-plaque transfer series. The initial population of bacteriophage $\mathrm{Q} \beta$ used for the present study had a long history of passages in the same biological environment, a fact that possibly allowed approaching a mutation-selection equilibrium. Plaque-to-plaque transfers may displace virus populations from mutation-selection equilibrium, due to the stochastic choice of the founder virus, and the random accumulation of mutations. In clonal populations that were close to a mutation-selection equilibrium ( $\mathrm{C} 1 \mathrm{pt} 1$ and $\mathrm{C} 2 \mathrm{pt} 1)$ an increase in error rate may generate beneficial mutations with low probability. In contrast, in populations which deviated from the mutation-selection equilibrium (C3pt38 and C4pt40) an increase in error rate may generate beneficial mutations with higher probability. In this view, the genomic context would become a highly relevant factor to determine whether a virus would gain or lose fitness as a consequence of the increase of the error rate. The observations with bacteriophage $\mathrm{Q} \beta$ agree with previous results showing that FMDV clones that had decreased their fitness as a consequence of a large number of plaque-toplaque transfers were more prone to acquire beneficial or compensatory mutations than high fitness viruses that were subjected to a lower number of transfers. ${ }^{30,41}$ 
Our model studies with bacteriophage $\mathrm{Q} \beta$ may be relevant to a lethal mutagenesis approach in vivo, because they suggest a limitation to the success of a mutagenesisbased therapy in the case of viruses that colonize organs or tissues, and that transmit from cell to cell under conditions of limited diffusion. Modifications of lethal mutagenesis protocols to try to anticipate these limitations are currently under study.

\section{Materials and Methods}

Viruses and bacteria. Standard procedures for infection.

Bacteriophage $\mathrm{Q} \beta$ was routinely propagated by infecting log-phase cultures of E. coli, strain Hfr (Hayes) in NB medium (2 g/l Nutrient Broth from Merck, $1.25 \mathrm{~g} / 1 \mathrm{NaCl}$ ). Both the phage and the bacteria were a generous gift of Dr C.K. Biebricher. The phage population was adapted to replication in liquid culture medium in our laboratory by infecting 1 liter of an exponential phase $E$. coli culture at a MOI of 1 pfu per bacterium. The culture was incubated at $37^{\circ} \mathrm{C}$ with good aeration during $90 \mathrm{~min}$ and at this point, an aliquot was used to infect another fresh exponential culture of E. coli at the same MOI. This process was repeated for a total of 5 serial transfers. The lysate at the fifth transfer was clarified by incubating with a few drops of chloroform for $30 \mathrm{~min}$ at room temperature with aeration, after which it was centrifuged for $40 \mathrm{~min}$ at $8000 \mathrm{x}$ g. Phages from the supernatant were purified by precipitation with $6 \%$ polyethylene glycol 6000 and differential sedimentation. The phage population obtained in this way had a titer, determined by plaque assay, of $1.3 \times 10^{11} \mathrm{pfu} / \mathrm{ml}$ and was stored in $50 \%(\mathrm{v} / \mathrm{v})$ glycerol in phage buffer (PB: $0.25 \mathrm{~g} / 1$ gelatine, $0.05 \mathrm{M}$ Tris- $\mathrm{HCl} \mathrm{pH} 7.5,0.01 \mathrm{M} \mathrm{MgCl}_{2}$ ) at $-80^{\circ}$ C. This population was used in all the experiments described in this study. 
Infections in liquid medium were always carried out using fresh exponential phase $E$. coli cultures $\left(\mathrm{OD}_{550 \mathrm{~nm}}\right.$ between 0.6 and 0.8$)$ that were infected with bacteriophage $\mathrm{Q} \beta$ at the MOI indicated for each experiment. At $2 \mathrm{~h}$ post infection, cultures were clarified upon treatment with chloroform. Virus supernatants were harvested by centrifugation at $13000 \mathrm{x} g$ for 15 minutes and maintained at $4^{\circ}$ for short-term use (less than 15 days) or at $-80^{\circ} \mathrm{C}$ for long-term storage.

Infections in semisolid agar were carried out by mixing $300 \mu 1$ of exponential phase bacteria with $100 \mu \mathrm{l}$ of phage in $3.5 \mathrm{ml}$ of melted top agar. The suspension was poured onto Petri dishes containing a bottom agar layer. The phage concentration was adjusted to obtain a number of lytic plaques between 20 and 40, to ensure that each plaque resulted from replication of a single virus particle. Development of lytic plaques in the presence of AZC was carried out by mixing the top agar with a solution of this compound to the desired final concentration, as indicated for each experiment. The volume of the bottom agar layer in the plaque assays carried out with AZC was always $20 \mathrm{ml}$.

Biological clones were isolated by punching and removing the top and bottom agar around well separated lytic plaques. The agar containing the lytic plaque was transferred into an eppendorf tube with $1 \mathrm{ml}$ of $\mathrm{PB}$ and $50 \mu \mathrm{l}$ of chloroform, and incubated for $1 \mathrm{~h}$ at $28^{\circ} \mathrm{C}$ with shaking (300 rpm). After centrifugation at $13000 \mathrm{x}$ g for $15 \mathrm{~min}$ to clarify the supernatant, the latter was stored over $50 \mu 1$ of chloroform at $4^{\circ} \mathrm{C}$ (to be used during a maximum of 2 weeks) or at $-80^{\circ} \mathrm{C}$. 
Plaque-to-plaque transfers were carried out by making serial infections at low MOI (about $10^{-7}$ pfu per bacterium) in semisolid agar, using the virus contained in a well isolated plaque obtained at the previous passage. The process was repeated the number of times indicated for each experiment. The virus present in each plaque was titrated at each transfer. The number of infectious particles per plaque was used as an estimate of the replicative ability of the founder virus.

Toxicity of AZC for E. coli

The effect of the mutagen AZC on the viability of $E$. coli growing in liquid medium was determined by incubating $1 \mathrm{ml}$ of an exponential phase culture with increasing AZC concentrations during $2 \mathrm{~h}$ at $37^{\circ} \mathrm{C}$. Both, the optical density at $550 \mathrm{~nm}$ and the number of colony-forming units were determined for each culture, and referred to the values obtained for a control culture that was treated in parallel without AZC. The results obtained indicated that $120 \mu \mathrm{g} / \mathrm{ml} \mathrm{AZC}$, which is the concentration used in most of the experiments, had an almost negligible effect on the viability of the E. coli strain used in this study. This AZC concentration neither affected the formation of a confluent lawn of the bacteria in semisolid agar.

Correlation between plaque titers and the absolute growth rate in infections in liquid medium

The same number of pfu $\left(5 \times 10^{3}\right)$ from 5 lytic plaques (with titers ranging from $5 \times 10^{4}$ to $1 \times 10^{8}$ pfu per plaque) was used to infect $E$. coli cultures in a volume of $1 \mathrm{ml}$. Viral supernatants were collected $2 \mathrm{~h}$ post infection and titrated. A significant linear 
correlation between the plaque titers and the virus yield obtained in the liquid medium cultures was observed (Pearson's correlation coefficient $r=0.99, p<0.05$ ).

Serial transfers of bacteriophage $Q \beta$ populations in the presence of $A Z C$

Serial transfers of bacteriophage $\mathrm{Q} \beta$ were carried out by infecting $1 \mathrm{ml}$ cultures of $E$. coli at the exponential phase with $4 \times 10^{7}$ pfu $(\mathrm{MOI} \approx 0.1$ pfu per bacterium). Phages were allowed to penetrate into the bacteria by incubating during $10 \mathrm{~min}$ at $37^{\circ} \mathrm{C}$ with low shaking $(100 \mathrm{rpm})$. The non-absorbed phages were eliminated by centrifugation at $13000 \mathrm{x} \mathrm{g}$ for $10 \mathrm{~min}$ at $4^{\circ} \mathrm{C}$. The pellet containing the bacteria with the adsorbed phages was resuspended in $1 \mathrm{ml}$ of NB containing the desired AZC concentration. After incubation for $2 \mathrm{~h}$ at $37^{\circ} \mathrm{C}$ with good aeration, the virus supernatants were clarified upon treatment with $50 \mu \mathrm{l}$ of chloroform, collected by centrifugation and $100 \mu 1$ of the phage suspension were used to initiate the infection of a fresh $E$. coli culture. The virus supernatant was titrated at each transfer to determine the phage yield. This protocol was slightly modified in the case of the experiments designed to compare the effect of AZC in liquid medium and in semisolid agar infections. The MOI was decreased to $10^{-7} \mathrm{pfu}$ per bacterium and the bacteria with the adsorbed phage were resuspended in a final volume of $3 \mathrm{ml}$.

RNA extraction, cDNA synthesis, PCR amplification, and nucleotide sequencing

RNA was extracted by treatment of the phage suspension with $1 / 10$ vol $10 \%$ SDS, $1 / 10$ vol $50 \% \beta$-mercaptoethanol and $1 \mu \mathrm{l}$ of RNAsine during $20 \mathrm{~min}$ at room temperature with low shaking. After $5 \mathrm{~min}$ in ice, the sample was extracted with cold water- 
saturated phenol and the upper phase was precipitated with ethanol following standard procedures. RNA samples were resuspended in RNAse-free water and stored at $-80^{\circ} \mathrm{C}$.

RNAs were amplified by RT-PCR using Avian Myeloblastosis Virus reverse transcriptase (Promega) and Expand High Fidelity DNA polymerase (Roche). The cDNAs were purified with a Wizard PCR purification kit (Promega), and subjected to cycle sequencing with Big Dye Chemistry (Applied Biosystems; Perkin-Elmer). The products were analyzed with an automated sequencer (Applied Byosystems; PerkinElmer). The following pairs of oligonucleotide primers were used for RT-PCR: P1 forward (5'CGAATCTTCCGACACGCATCC3') with P1 reverse (5'AAACGGTAACACTTCTCCAG3') to amplify from nucleotide position 148 to 1497; P2 forward (5'CTCAATCCGCGTGGGGTAAATCC3') with P2 reverse (5'CAGAAAATCGGCAGTGACGCAACA3') to amplify from nucleotide position 1407 to 2817; and P3 forward (5'-CCGATGGCGTGATAGTTG3') with P3 reverse (5'TCGTGCCCTGGAAGAGG-3') to amplify from nucleotide position 2243 to 4095. Sequences were aligned with the consensus sequence of wt phage with Clustal W. Mutations relative to the consensus sequence were identified using the program BioEdit.

\section{Acknowledgments}

We are indebted to Professor C. K. Biebricher for many useful discussions thorough the development of this study, and to S. C. Manrubia for the critical reading of the manuscript. Work at $\mathrm{CAB}$ was supported by the Instituto Nacional de Técnica Aeroespacial (INTA) and by the Spanish Ministerio de Educación y Ciencia through project FIS6404-2004. CIBERehd is funded by the Instituto de Salud Carlos III. 


\section{References}

1. Batschelet, E., Domingo, E. \& Weissmann C. (1976). The proportion of revertant and mutant phage in a growing population, as a function of mutation and growth rate. Gene $1,27-32$.

2. Drake, J. W. (1993). Rates of spontaneous mutation among RNA viruses. Proc. Natl. Acad. Sci. USA 90, 4171-4175.

3. Domingo, E. \& Holland, J. J. (1997). RNA virus mutations and fitness for survival. Annu. Rev. Microbiol. 51, 151-178.

4. Drake, J. W. \& Holland, J. J. (1999). Mutation rates among RNA viruses. Proc. Natl. Acad. Sci. USA 96, 13910-13913.

5. Eigen, M. (1971). Selforganization of matter and the evolution of biological macromolecules. Naturwissenschaften 58, 465-523.

6. Eigen, M., \& Schuster, P. (1979). The Hypercycle. A principle of Natural SelfOrganization. Springer Verlag, Berlin.

7. Saakian, D. B \& Hu, C. K. (2006). Exact solution of the Eigen model with general fitness functions and degradation rates. Proc. Natl. Acad. Sci. USA 103, 4935-4939. 
8. Eigen, M., Mc CasKill, J. \& Schuster, P. (1988). Molecular Quasi-species. J. Phys. Chem. 92, 6881-6891.

9. Biebricher, C. K. \& Eigen, M. (2006). What is a quasispecies? Curr. Top. Microbiol. Immunol. 299, 1-31.

10. Swetina, J. \& Schuster, P. (1982). Self-replication with errors. A model for polynucleotide replication. Biophys. Chem. 16, 329-345.

11. Eigen, M. (2002). Error catastrophe and antiviral strategy. Proc. Natl. Acad. Sci. USA $99,13374-13376$.

12. Biebricher, C. K. \& Eigen, M. (2005). The error threshold. Virus Res. 107, 117-127.

13. González-López, C., Gómez-Mariano, G., Escarmís, C. \& Domingo, E. (2005). Invariant aphthovirus consensus nucleotide sequence in the transition to error catastrophe. Infect. Genet. Evol. 5, 366-374.

14. Grande-Pérez, A., Lázaro, E., Lowenstein, P., Domingo, E. \& Manrubia, S. C. (2005). Suppression of viral infectivity through lethal defection. Proc. Natl. Acad. Sci. USA $102,4448-4452$.

15. Loeb, L. A., Essigmann, J. M., Kazazi, F., Zhang, J., Rose, K. D. \& Mullins, J. I. (1999). Lethal mutagenesis of HIV with mutagenic nucleoside analogs. Proc. Natl. Acad. Sci. USA 96, 1492-1497. 
16. Anderson, J. P., Daifuku, R. \& Loeb L. A. (2004). Viral error catastrophe by mutagenic nucleosides. Annu. Rev. Microbiol. 58, 183-205.

17. Graci, J. D. \& Cameron, C.E. (2006). Mechanisms of action of ribavirin against distinct viruses. Rev. Med. Virol. 16, 37-48.

18. Domingo, E., Escarmís, C., Lázaro, E. \& Manrubia, S. C. (2005). Quasispecies dynamics and RNA virus extinction. Virus Res. 107, 129-139.

19. González-López, C., Gómez-Mariano, G. Escarmís, C. \& Domingo, E. (2004). Preextinction viral RNA can interfere with infectivity. J. Virol. 78, 3319-3324.

20. Perales, C., Mateo, R., Mateu, M. G. \& Domingo, E. (2007). Insights into RNA virus mutant spectrum and lethal mutagenesis events: replicative interference and complementation by multiple point mutants. J. Mol. Biol. 369, 985-1000.

21. Escarmís, C., Lázaro, E., Arias, A. \& Domingo, E. (2008). Repeated bottleneck transfers can lead to non-cytocidal forms of a cytopathic virus: implications for viral extinction. J. Mol. Biol. 376, 367-379.

22. Novella, I. S., Elena, S. F., Moya, A., Domingo, E. \& Holland, J. J. (1995). Size of genetic bottlenecks leading to virus fitness loss is determined by mean initial population fitness. J. Virol. 69, 2869-2872. 
23. Chao, L. (1990). Fitness of RNA virus decreased by Muller's ratchet. Nature 348, 454-455.

24. Duarte, E., Clarke, D., Moya, A., Domingo, E. \& Holland, J. (1992). Rapid fitness losses in mammalian RNA virus clones due to Muller's ratchet. Proc. Natl. Acad. Sci. USA $89,6015-6019$.

25. Escarmís, C., Dávila, M., Charpentier, N., Bracho, A., Moya, A. \& Domingo, E. (1996). Genetic lesions associated with Muller's ratchet in an RNA virus. J. Mol. Biol. $264,255-267$.

26. Yuste, E., Sánchez-Palomino, S., Casado, C., Domingo, E. \& López-Galíndez, C. (1999). Drastic fitness loss in human immunodeficiency virus type 1 upon serial bottleneck events. J. Virol. 73, 2745-2751.

27. de la Iglesia, F. \& Elena, S. F. (2007). Fitness Declines in Tobacco Etch Virus upon Serial Bottleneck Transfers. J. Virol. 81, 4941-4947.

28. Martin, G. \& Lenormand, T. (2006). The fitness effect of mutations across environments: a survey in light of fitness landscape models. Evolution Int. J. Org Evolution 60, 2413-2427.

29. Escarmís, C., Lázaro, E. \& Manrubia, S. C. (2006). Population bottlenecks in quasispecies dynamics. Curr. Top. Microbiol. Immunol. 299, 141-170. 
30. Escarmís, C., Gómez-Mariano, G., Dávila, M., Lázaro, E. \& Domingo, E. (2002). Resistance to extinction of low fitness virus subjected to plaque-to-plaque transfers: diversification by mutation clustering. J. Mol. Biol. 315, 647-661.

31. Lázaro, E., Escarmís, C., Domingo, E. \& Manrubia, S. C. (2002). Modeling viral genome fitness evolution associated with serial bottleneck events: evidence of stationary states of fitness. J. Virol. 76, 8675-8681.

32. Manrubia, S. C., Escarmís, C., Domingo, E. \& Lázaro, E. (2005). High mutation rates, bottlenecks, and robustness of RNA viral quasispecies. Gene 347, 273-282.

33. Overby, L. R, Barlow, G. H., Doi, R. H., Jacob, M. \& Spiegelman S. (1966). Comparison of two serologically distinct ribonucleic acid bacteriophages. I. Properties of the viral particles. J. Bacteriol. 91, 442-448.

34. Domingo, E., Sabo, D., Taniguchi, T. \& Weissmann, C. (1978). Nucleotide sequence heterogeneity of an RNA phage population. Cell 13, 735-744.

35. Domingo, E., Flavell, R. A. \& Weissmann, C. (1976). In vitro site-directed mutagenesis: generation and properties of an infectious extracistronic mutant of bacteriophage Qbeta. Gene 1, 3-25.

36. Biebricher, C. K. (2008). Mutation, competition, and selection as measured with small RNA molecules. In Origin and evolution of viruses. $2^{\text {nd }}$ edition (Domingo, E., Parrish, C. R. and Holland, J. J. eds.) Elsevier, Oxford, pp. 65-86. 
37. Halle, S. (1968). 5-Azacytidine as a mutagen for arboviruses. J. Virol. 2, 1228-1229.

38. Holland, J. J., Domingo, E., de la Torre, J. C. \& Steinhauer, D. A. (1990). Mutation frequencies at defined single codon sites in vesicular stomatitis virus and poliovirus can be increased only slightly by chemical mutagenesis. J. Virol. 64, 3960-3962.

39. Lee, C. H., Gilbertson, D. L., Novella, I. S., Huerta, R., Domingo, E. \& Holland, J. J. (1997). Negative effects of chemical mutagenesis on the adaptive behavior of vesicular stomatitis virus. J. Virol. 71, 3636-3640.

40. Crotty, S., Cameron, C. E. \& Andino R. (2001) RNA virus error catastrophe: direct molecular test by using ribavirin. Proc. Natl. Acad. Sci. USA. 98, 6895-6900.

41. Lázaro, E., Escarmís, C., Pérez-Mercader, J., Manrubia, S. C., and Domingo, E. (2003). Resistance of virus to extinction on bottleneck passages: study of a decaying and fluctuating pattern of fitness loss. Proc. Natl. Acad. Sci. USA. 100, 10830-10835.

42. Horiuchi, K. (1975). Genetic studies of RNA phages. In: RNA Phages, N.D. Zinder, ed., Cold Spring Harbor Laboratory pp. 29-50.

43. Grande-Pérez, A., Sierra, S., Castro, M. G., Domingo, E. \& Lowenstein, P. R. (2002). Molecular indetermination in the transition to error catastrophe: systematic elimination of lymphocytic choriomeningitis virus through mutagenesis does not 
correlate linearly with large increases in mutant spectrum complexity. Proc. Natl. Acad. Sci. USA 99, 12938-12943.

44. Tapia, N., Fernández, G., Parera, M., Gómez-Mariano, G., Clotet, B., QuiñonesMateu, M., Domingo, E. \& Martínez, M. A. (2005). Combination of a mutagenic agent with a reverse transcriptase inhibitor results in systematic inhibition of HIV-1 infection. Virology. 338, 1-8.

45. Aguirre, J. \& Manrubia, S. C. (2008). Effects of spatial competition on the diversity of a quasispecies. Phys. Rev. Lett. 100, 038106.

46. Yin, J. \& McCaskill, J. S. (1992). Replication of viruses in a growing plaque: a reaction-diffusion model. Biophys J. 61, 1540-1549.

47. Yin, J. (1993). Evolution of bacteriophage T7 in a growing plaque. J. Bacteriol. 175, $1272-1277$

48. Sanjuán, R., Moya, A. \& Elena, S. F. (2004). The contribution of epistasis to the architecture of fitness in an RNA virus. Proc. Natl. Acad. Sci. USA 101, 15376-15379.

49. Parera, M., Fernández, G., Clotet, B. \& Martínez, M. A. (2007). HIV-1 protease catalytic efficiency effects caused by random single amino acid substitutions. Mol. Biol. Evol. 24, 382-387. 


\section{Legend to figures.}

Fig. 1. Virus yields obtained upon subjecting a population of bacteriophage Q $\beta$ to serial passages in E. coli. Passages were carried out either in the absence (control, C) or presence of increasing concentrations of AZC (40, 80, and $120 \mu \mathrm{g} / \mathrm{ml})$. At each passage, $100 \mu 1$ of the phage population from the previous transfer was used to infect a fresh E. coli culture, under the conditions described in Materials and Methods.

Fig. 2. Titers of the lytic plaques of the plaque-to-plaque transfer series carried out starting with virus clones $\mathrm{C} 3 \mathrm{pt} 1$ and $\mathrm{C} 4 \mathrm{pt} 1$ in the absence of AZC. Procedures are detailed in Materials and Methods.

Fig. 3. Titer of the subclones isolated upon plating virus clones $\mathrm{C} 1 \mathrm{pt} 1, \mathrm{C} 2 \mathrm{pt} 1, \mathrm{C} 3 \mathrm{pt} 38$, and C4pt40 either in the absence or the presence of AZC $(120 \mu \mathrm{g} / \mathrm{ml})$. The points represent the titer for each individual subclone. The dashed line indicates the titer of the corresponding parental clonal population and the solid lines represent the average titer for all the subclones isolated from each parental clone. The statistical significance for the difference of means obtained with and without AZC is also indicated ( $p$ value, Student's $t$ test).

Fig. 4. Scheme of isolation of subclones from clone $\mathrm{C} 4 \mathrm{pt} 40$, and average titers after various treatments. Procedures are detailed in Materials and Methods. 
Fig. 5. Serial passages in liquid medium and plaque-to-plaque transfers of virus clones C4pt41.16 and C4pt41.17 either in the absence or the presence of AZC $(120 \mu \mathrm{g} / \mathrm{ml})$. Procedures are detailed in Materials and Methods.

Fig. 6. Scheme showing the origin and the evolution of the titers of two clones isolated from preextinction populations, and subjected to three additional plaque-to-plaque transfers in the presence of AZC. Procedures are detailed in Materials and Methods. 
Table 1. Mutation frequency in biological clones isolated in liquid culture medium infections, carried out in the presence of different AZC concentrations.

\begin{tabular}{|c|c|c|c|}
\hline $\begin{array}{l}\text { AZC concentration } \\
\qquad(\mu \mathrm{g} / \mathrm{ml})^{\mathrm{a}}\end{array}$ & $\begin{array}{l}\text { Passage } \\
\text { number }^{\mathrm{a}}\end{array}$ & $\begin{array}{l}\text { Number of clones } \\
\text { sequenced }\end{array}$ & Mutation frequency ${ }^{b}$ \\
\hline 0 & 3 & 8 & $9.8 \times 10^{-5}$ \\
\hline 40 & 3 & 10 & $7.6 \times 10^{-4}(p<0.001)$ \\
\hline 80 & 3 & 9 & $9.2 \times 10^{-4}(p<0.001)$ \\
\hline 120 & 3 & 9 & $5.0 \times 10^{-4}(p=0.07)^{*}$ \\
\hline 0 & 6 & 16 & $3.7 \times 10^{-4}$ \\
\hline 40 & 6 & 10 & $1.2 \times 10^{-3}(p=0.01)$ \\
\hline 80 & 6 & 7 & $7.3 \times 10^{-4}(p=0.09)$ \\
\hline 120 & 6 & Extinguished & - \\
\hline 0 & 13 & 8 & $5.7 \times 10^{-4}$ \\
\hline 40 & 13 & 12 & $1.3 \times 10^{-3}(p=0.17)$ \\
\hline 80 & 13 & Extinguished & - \\
\hline 120 & 13 & Extinguished & - \\
\hline
\end{tabular}

${ }^{a}$ The cultures from which the biological clones were isolated are those described in Fig.1. A Student's $t$ test was used to analyze the statistical significance of the difference of means between the untreated controls and the samples treated with AZC. The asterisk indicates the transfer corresponding to a preextinction population which is defined as the last population from which infectivity could still be derived. Dash indicates a population which was extinguished and for which no RNA could be amplified. 
${ }^{b}$ Mutation frequencies were calculated by dividing the number of different mutations found (relative to the consensus sequence of the parental population) by the total number of nucleotides sequenced ( $\mathrm{Q} \beta$ genomic residues 1407 to 2817). 
Table 2. Mutations fixed in the genomes of virus clones C3pt40 and C4pt40

\begin{tabular}{ll}
\hline C3pt40 & C4pt40 \\
\hline C360T & A469G \\
A909G & A861G \\
T987G & T1942C \\
C1194A & A2557G \\
T1418C & A2656G \\
G1542A & G3609A \\
T2515C & G3629A \\
T2526C & A3912G \\
T2527C & \\
\hline
\end{tabular}

${ }^{\mathrm{a}}$ Genomic residues are numbered according to the consensus sequence of the parental population. Procedures for nucleotide sequence determination are described in Materials and Methods. 
Figure 1

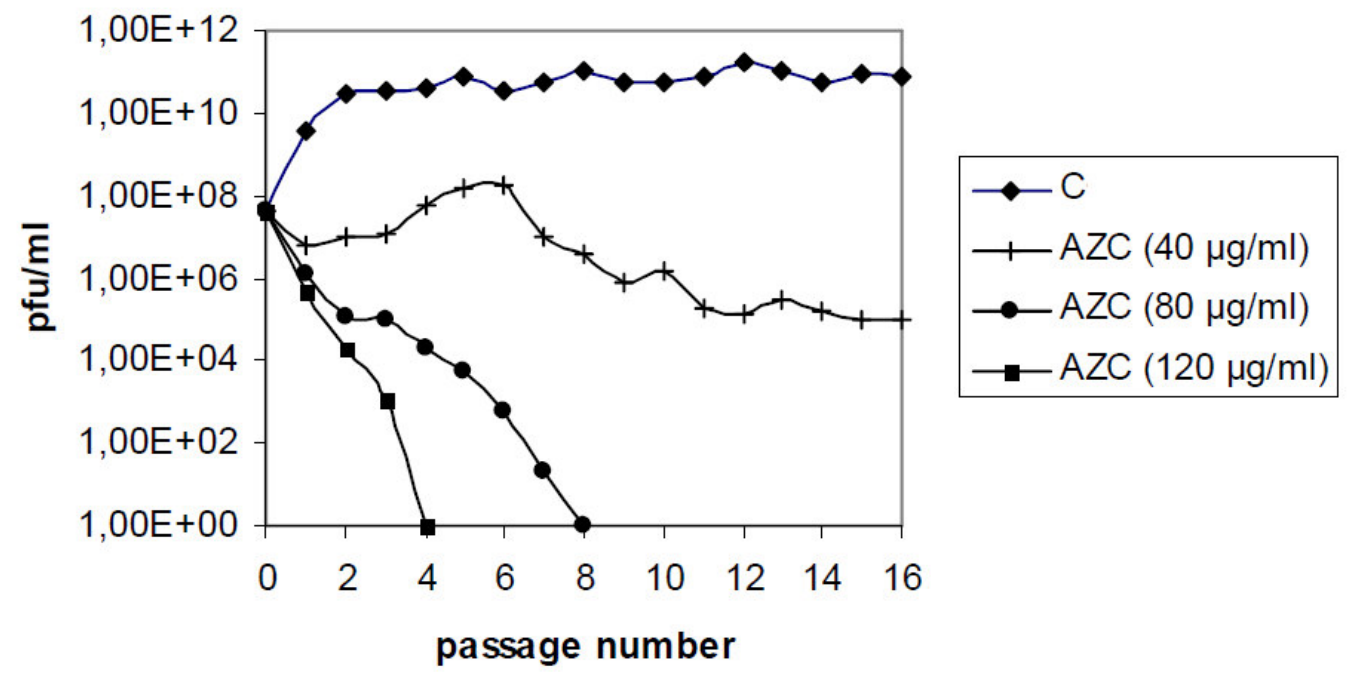


Figure 2

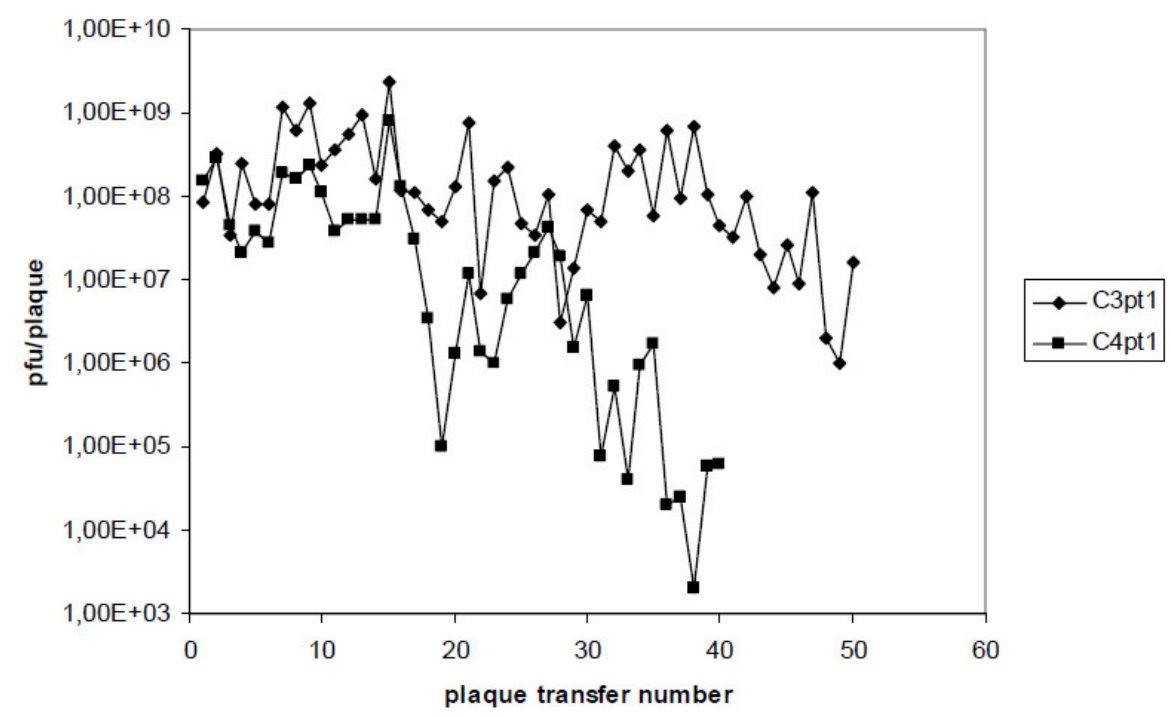


Figure 3

C1pt1 subclones

C2pt1 subclones
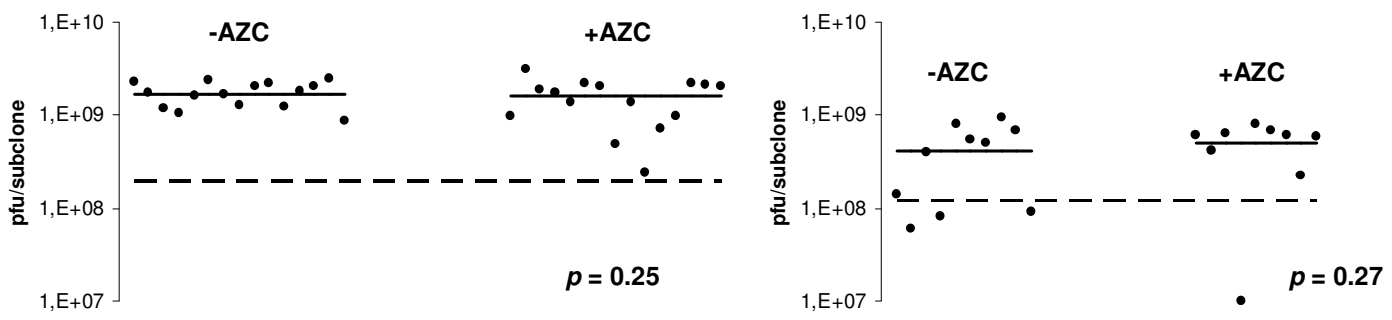

C3pt38 subclones
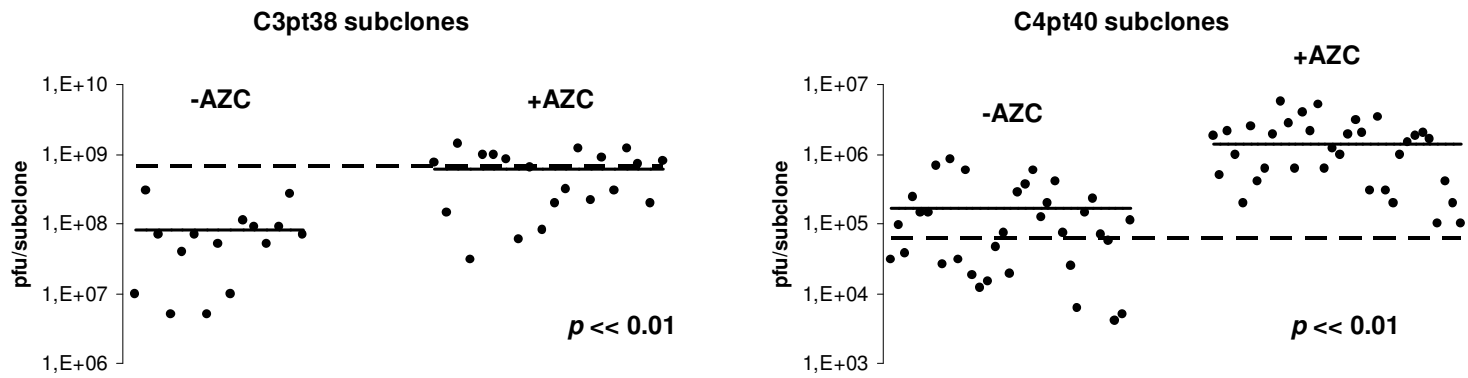
Figure 4

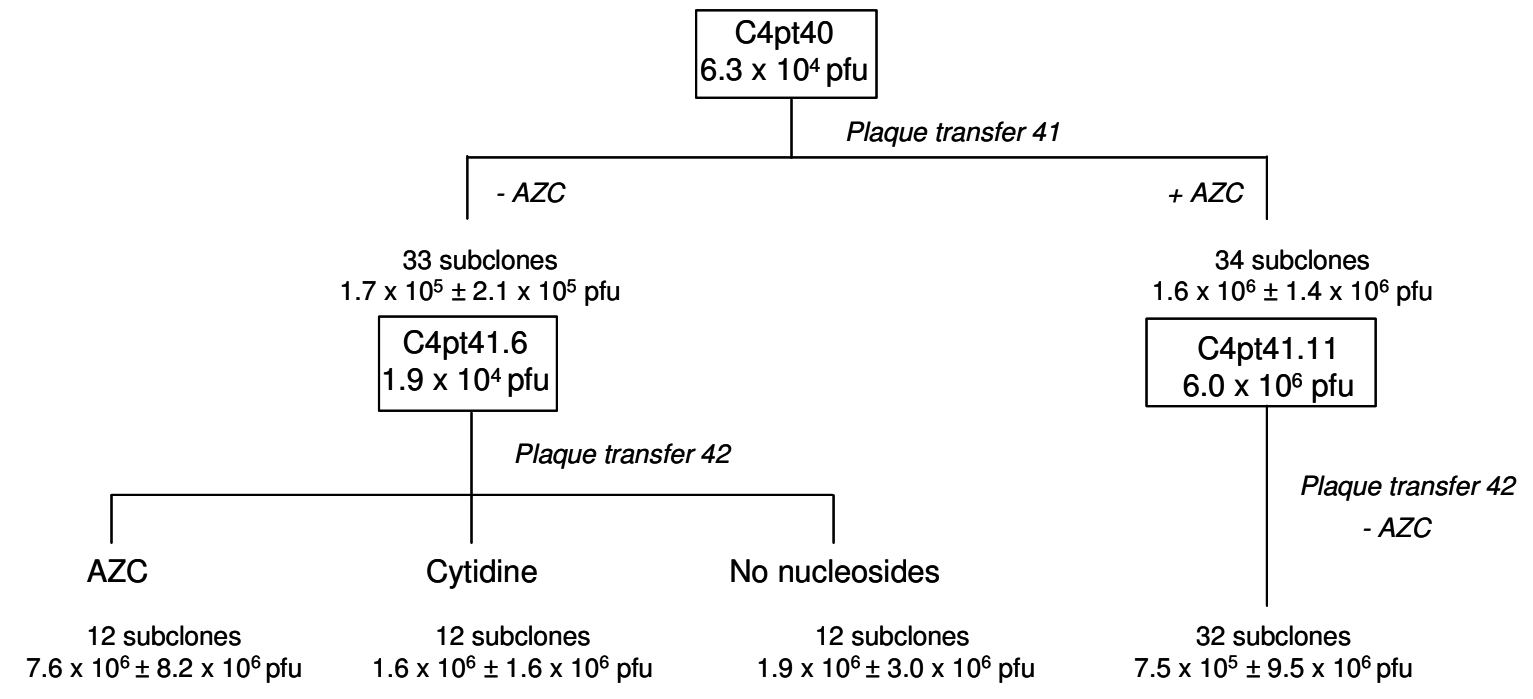


Figure 5
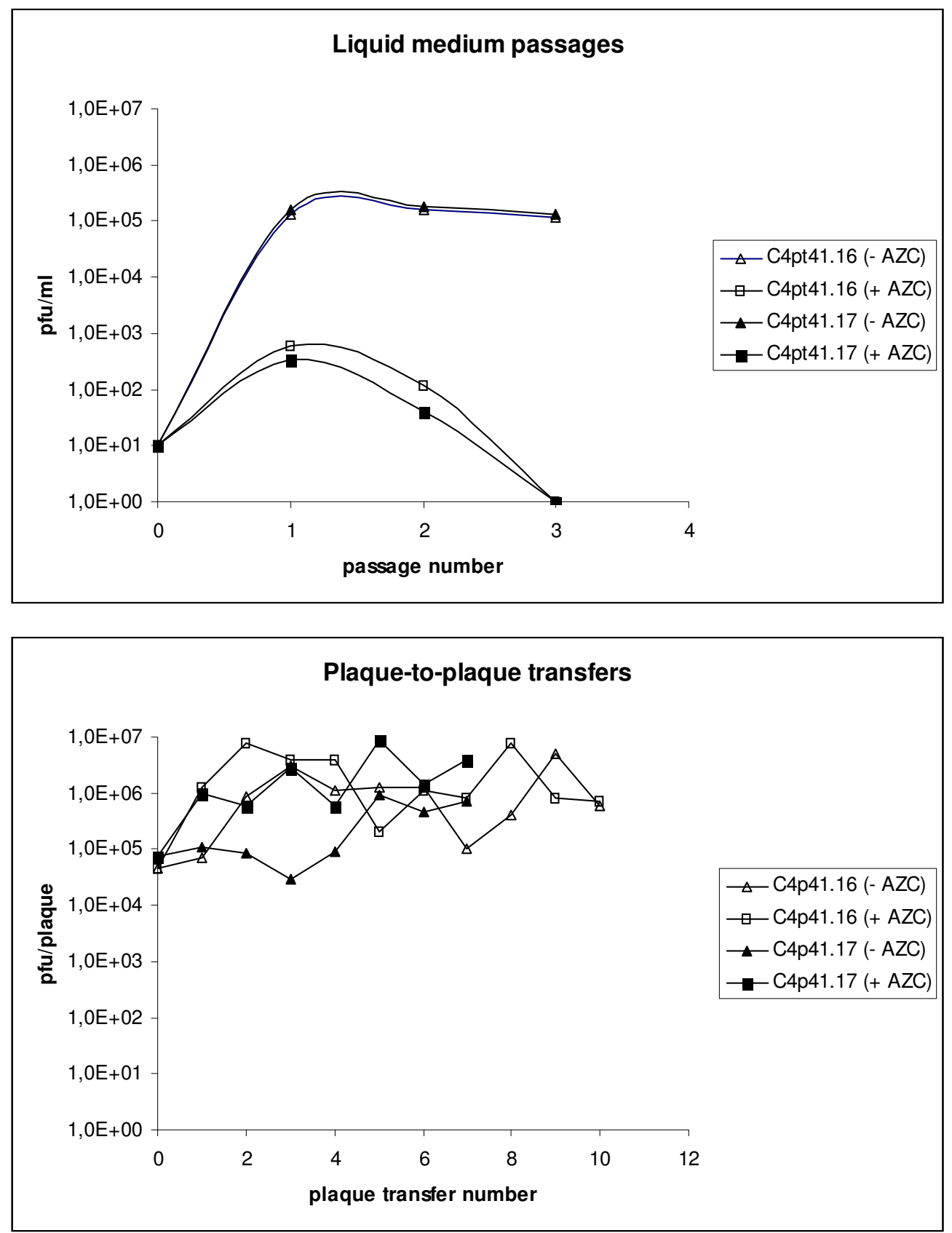
Figure 6

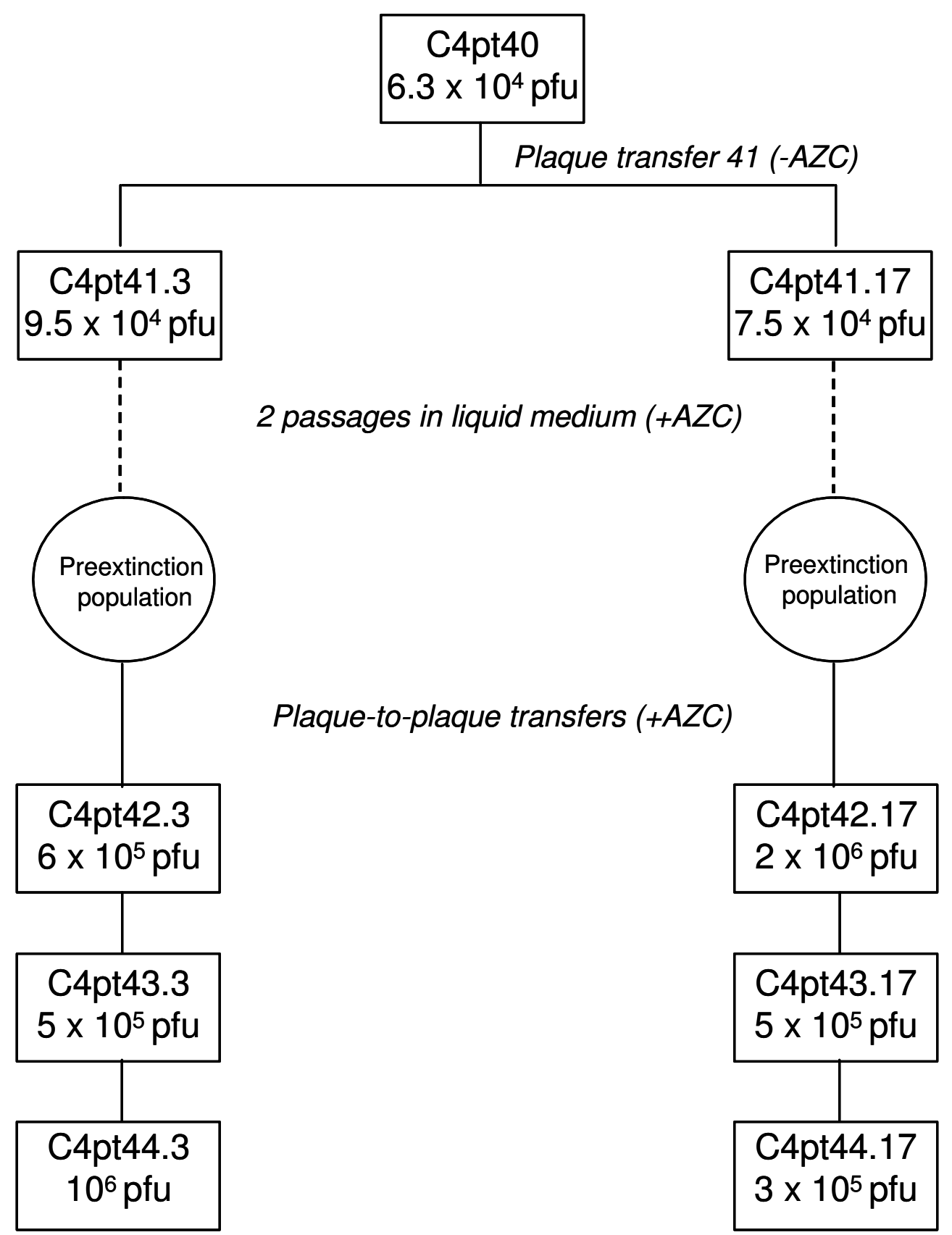

